\title{
On Spectral Analysis of Heart Rate Variability during Very Slow Yogic Breathing
}

\author{
E. Jovanov \\ Electrical and Computer Engineering Department \\ University of Alabama in Huntsville, Huntsville, Alabama, U.S.A.
}

\begin{abstract}
Very slow yogic breathing techniques provide valuable insights into mechanisms of autonomous nervous system regulation that are usually not available for human subjects. This paper presents results of eight sessions of Nadi Shodhana Pranayama practiced at rate of one breath per minute. We characterized statistic and spectral measures of heart rate variability before, during, and after exercises. Significant changes include increase of VLF frequencies caused by slow breathing and decrease in average interbeat interval from 959.3 to $904.1 \mathrm{~ms}(\mathrm{t}(7)=-7.5, p<0.001)$. We present the results of HRV analysis and analyze origins of characteristic frequency components. The most prominent changes of the exercise include significant increase of respiratory sinus arrhythmia (RSA) and LF/HF ratio, and decrease of breathing frequency after the exercise against the state before the exercise. The maximum LF frequency decreased from $0.0919 \mathrm{~Hz}$ to $0.07125 \mathrm{~Hz}(\mathrm{t}(7)=-\mathbf{3 . 2 5 5}, \mathrm{p}$ $<0.01$ ), indicating the decrease of average breathing rhythm from 5.5 breaths $/ \mathrm{min}$ to $4.3 \mathrm{breaths} / \mathrm{min}$. In addition, the state after the exercise is characterized by disappearance of VLF frequencies from the spectrum, and a significant increase of $\mathrm{LF} / \mathrm{HF}$ from 14.33 to $\mathbf{5 0 . 9 3}$ $(t(7)=2.461, p<.05)$.
\end{abstract}

Keywords - Heart Rate Variability, Respiratory Sinus Arrhythmia, Yogic Breathing, Spectral Analysis, Cardiorespiratory Interaction

\section{INTRODUCTION}

Respiratory sinus arrhythmia (RSA) is a change of heart rate generated by a combination of respiration-induced biochemical changes, changes in intrathoracic pressure, and central vagal stimulation [1][2]. Baroreflex sensitivity (BRS) and heart rate variability (HRV) are used to assess the state of autonomous nervous system (ANS) for diagnostic and prognostic purposes, as well as for biofeedback training. Frequency of RSA component falls in High Frequency range of HRV $(0.15-0.4 \mathrm{~Hz})$ for more than 9 breaths $/ \mathrm{min}$. Slower breathing generates peak power of HRV in Low Frequency (LF) range (0.04-0.15 Hz). Vaschillo et al. found that the highest oscillation amplitudes are measured in the range of $0.055-0.11 \mathrm{~Hz}$ and explained this as a resonant effect [3]. Song and Lehrer found that the respiration rate of 4 breaths $/ \mathrm{min}$ produced the highest amplitudes of HRV, while even lower rates ( 3 breaths/min) generated smaller amplitude [2].

It is very hard to characterize cardiac variability of human subjects in extremely low breathing frequencies, although they provide valuable characterizations of ANS activity. Very slow breathing techniques offer rare insights into mechanisms of ANS regulation by decreasing frequency of RSA [4][5]. Very slow breathing with frequency of less than $0.04 \mathrm{~Hz}$ (more than 25 seconds/breath) generates a dominant respiration component in Very Low Frequency (VLF) band $(0.003-0.04 \mathrm{~Hz})$. Our preliminary study overemphasized the importance of high frequencies that were produced by several arrhythmic events [5]. Therefore, we decided to characterize very slow breathing in strictly controlled experimental settings and with larger number of experiments. This paper presents analysis of HRV during very slow yogic breathing at one breath/min and explains origins of specific frequency components in HRV spectrum.

\section{METHODS}

This paper presents spectral analysis of HRV taken from eight measurements on a single practitioner during a period of six months. We measured heart rate variability using the WHRM system because of precise time stamps and possible synchronization with events [6]. Heart rate was monitored with $1 \mathrm{~ms}$ resolution.

The correctness of the heart rate variability signal was tested using a DelMar PWA holter amplifier and manual annotation. This was particularly important for the analysis of ectopic beats to exclude possible measurement errors. Breathing was monitored using the NIMS Respitrace 200 data acquisition system that provides four signals: chest volume, abdominal volume, sum (total volume), and flow. All four signals were sampled at $250 \mathrm{~Hz}$ and synchronized with ECG measurements. All ectopic beats create high frequency oscillations that could be filtered out [7]; however, this study presents the results of unmodified HRV signal with all ectopic events. Blood oxygen saturation was measured in real time during practice using the ARES Unicorder system from Advanced Brain Monitoring [8].

Yogic breathing combines abdominal, thoracic, and clavicular breathing phases to maximize breathing volume and increase oxygen uptake [9]. The practitioner was a 44 year old healthy male, with 10 years of Yoga practice. All measurements were made during Nadi Shodhana Pranayama, or alternate nostril breathing. This technique encompasses four breathing phases: inhalation, internal retention (keeping lungs full), exhalation, and external retention (keeping lungs empty). The duration of breathing phases is controlled by mental counting in the ratio of $1: 2: 2: 1$. Inhalation and exhalation take place through 
altering active nostril, keeping the other nostril closed. Traditionally, the right nostril is closed by the thumb and left nostril is closed with the ring finger. For example, during the first cycle the practitioner would inhale through the left nostril, keeping right nostril closed, than keep the breath in ("internal retention") with both nostrils closed, exhale through right nostril only, and finally close both nostrils during external retention. The next cycle starts by inhaling through the right nostril. The practice consists of ten cycles of slow breathing as described above. Our subject was comfortable with the rate of one breath per minute, which means inhalation $(10 \mathrm{sec})$, internal retention $(20 \mathrm{sec})$, exhalation $(20 \mathrm{sec})$, and external retention $(10 \mathrm{sec})$. It is important to emphasize that the practitioner started his practice at the rate of four breaths $/ \mathrm{min}$, and reached one breath/min after one year of practicing and gradual increase of the cycle length.

The practitioner was in Siddhasana pose before, during, and after exercise [9]. This is a sitting pose with straight spine and crossed legs. Before and during exercise the practitioner didn't talk or move. It should be noted that in 12 minutes after this pose has been taken, the breathing would deepen, and HRV of the practitioner would be changed significantly in every experiment, as represented in Fig. 1a.

In the first two experiments the practitioner was asked to synchronize breathing with the watch in 10-20-20-10 second rhythm. Although it was very convenient from the signal processing perspective, we concluded that it generates too much strain and distraction for the practitioner. Therefore, the remaining sessions were not externally synchronized. We observed that those sessions featured even longer breathing periods without strain.

We analyzed heart rate variability for exactly five minutes before and after each exercise and throughout the exercise, independent of the length of the exercise. The average length of an exercise was $590.38 \mathrm{~s}(\sigma=28.76 \mathrm{~s})$ for 10 breathing cycles; therefore, the average breath duration during exercises was 59 seconds. One minute before and after each exercise was excluded from the analysis, since preparation for the exercise and return to normal position influenced typical HRV. Spectral and statistical analyses were performed using Nevrokard 6.4.0 and SPSS 11.5. The spectral analysis was performed using Hanning window with 512 seconds during exercise (resolution $0.001953 \mathrm{~Hz}$ ), and 256 seconds before and after exercise (resolution $0.003906 \mathrm{~Hz})$.

\section{RESULTS AND CONCULSIONS}

One cycle of slow yogic breathing is presented in Fig. 2 with symbolic representation of breathing phases. According to the HRV changes, breathing phases could be divided into the following HRV phases:
1. Inhalation (0-12s) phase has two sub phases:

1.a. Abdominal inhalation (0-5.5s) stimulates vagus and rapidly changes heart rate.

1.b. Thoracic and clavicular inhalation phases (5.512s) involve additional effort and create an increase of heart rate and a sympathetic burst. Without this phase heart rate would have fallen exponentially to the rate at the beginning of the phase 2 at $\mathrm{t}=12 \mathrm{~s}$.

2. Internal retention (12-30s) produces only small variations of $\mathrm{HRV}$, mostly in LF band. Average heart rate in this phase is around 55 beats/min, with minimum measured in all eight experiments of 46.2 beats $/ \mathrm{min}$.

3. Exhalation (30-50s) clearly exhibits two different subphases:

3.a. Clavicular exhalation followed by thoracic exhalation phases (30-43s) are very similar to internal retention phase described above.

3.b. Abdominal exhalation (43-50s) generates rapid increase of heart rate.

4. External retention (50-60s) continues to increase heart rate due to "negative pressure" to baroreceptors and change of blood oxygen level (it has been more than 40s without fresh air in lungs at the beginning of this phase).

According to this analysis, we can divide HRV changes during one breath cycle to three different HRV phases, as presented in Fig. 2:

Phase I consists of phases 3.b, 4., and 1.a which create the most prominent change of HRV during period of $\mathrm{T}_{\mathrm{I}} \approx 22 \mathrm{~s}$ (equivalent to $\mathrm{f}_{\mathrm{I}}=0.045 \mathrm{~Hz}$ ).

Phase II is the phase 1.b, characterized by sympathetic burst with period of $\mathrm{T}_{\text {II }} \approx 6 \mathrm{~s}$ (equivalent to $\mathrm{f}_{\mathrm{II}}=0.16 \mathrm{~Hz}$ ). Please note that this fact explains $\mathrm{HF}$ maximum frequency during exercise in Table I.

Phase III consists of phases 2. and 3.a and creates a "quiet" period without RSA that could be used for ANS characterization in the absence of RSA. Total length of this period is $\mathrm{T}_{\mathrm{III}} \approx 31 \mathrm{~s}$ (equivalent to $\mathrm{f}_{\mathrm{I}}=0.032 \mathrm{~Hz}$ ).

Our analysis provides guidance for assessment of spectral parameters of HRV and characterization of ANS. The experiments did not provide conclusive evidence for characterization of ANS during Phase III.

Average heart rate during exercises increased significantly from $62.55 \mathrm{bpm}$ to $66.36 \mathrm{bpm}(t(7)=7.5$, $p<0.001)$, and then decreased to $60.1 \mathrm{bpm}$ after exercise $(t(7)=-3.77, p<0.005)$. It is interesting that the change of total HRV power did not change significantly during $(t(7)=$ $-1.3)$ and after the exercise $(t(7)=-0.6)$. The main reason for that is that dramatic changes of heart rate during inhalation and exhalation are followed by a relatively "quiet" period during internal retention and start of exhalation (Fig. 1.b). 

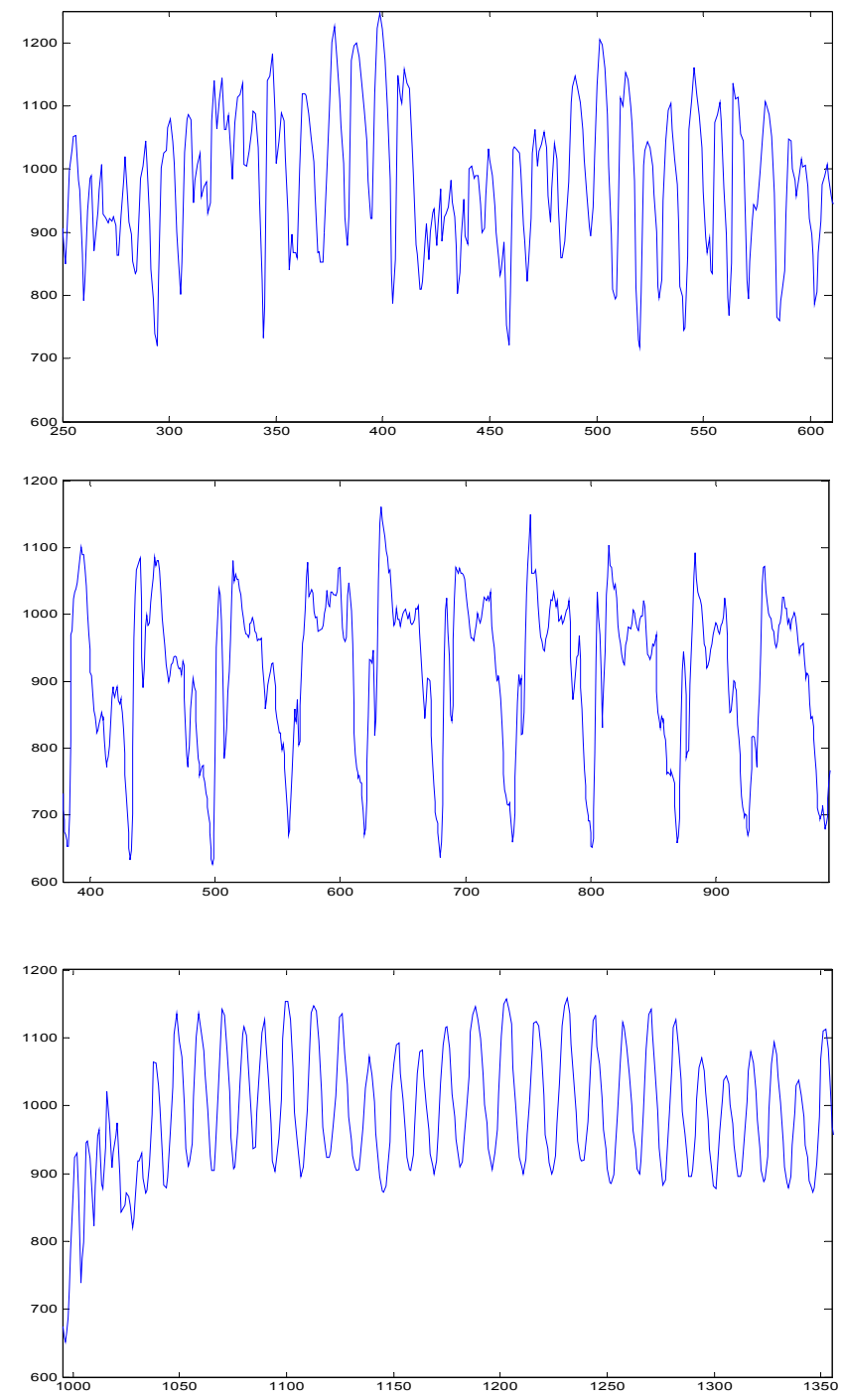

Fig. 1. HRV signal before (a), during (b), and after (c) very slow yogic breathing. During preparation for slow breathing amplitude of breathing increases and frequency decreases (file E01). In b) ten cycles of slow breathing are presented (file E04, $\mathrm{t}=378.5-988.5 \mathrm{~s}$, total length $610 \mathrm{~s}$, average $61 \mathrm{~s} /$ breath). Period of spontaneous breathing after exercise is given in (c) (file E06, length $6 \mathrm{~min}$ ). Please note establishment of a very large amplitude RSA component approximately 40 seconds after the end of the exercise, $\mathrm{LF} / \mathrm{HF}$ ratio during 5 minutes after exercise in this recording is 146 , with maximum power at frequency $\mathrm{f}=0.074 \mathrm{~Hz}$ ( 4.4 breaths $/ \mathrm{min}$ on the average).

Very slow breathing creates a significant change in the ratio of HRV frequency bands during and after the exercise. The most prominent changes are the change of relative VLF power before exercise $(\mathrm{M}=21.71 \%, \mathrm{SE}=3.48)$ and during exercise $\quad(\mathrm{M}=81.96 \%, \quad \mathrm{SE}=1.67), \quad t(7)=17.21, \quad p<.001$. Relative power of VLF band is significantly smaller after exercise $(\mathrm{M}=7.7 \%, \mathrm{SE}=3.76), t(7)=-4.02, p<.005$. Please note from Fig. 1.a that the "normal" HRV signal before the exercise is already changed very quickly after assuming the position, probably as a result of conditioning after years of practice. This makes the state before the exercise more similar to the state after exercise, therefore reducing the effect of the exercise. The main difference is longer period of HRV dominated by RSA component as it can be seen in Fig. 1.c.

Changes in VLF bands during exercise can be easily explained since the RSA frequency during exercise is in VLF band $(0.016 \mathrm{~Hz})$. However, significant changes in HF band are surprising. HF power is significantly reduced during exercise $t(7)=-5.04, p<.01$, and remains small after exercise $t(7)=-4.37, p<.002$. Ratio of LF and HF band is also significantly changed after exercise compared with the state before exercise, from 14.33 to $50.93, t(7)=2.461$, $p<.05$. The same ratio is not significantly changed during exercise.

Period after the exercise is characterized by the decrease of maximum LF frequency from $0.0919 \mathrm{~Hz}$ to $0.07125 \mathrm{~Hz}$ $(t(7)=-3.255, p<.01)$ that indicates decrease of average breathing rhythm from 5.5 breaths/min before exercise to 4.3 breaths/min after exercise.

We measured significant change of blood oxygen saturation. The value of $\mathrm{SpO} 2$ decreased from $98 \%$ before exercise in each breath cycle, reaching minimum of $83 \%$ in the fifth cycle, but recovering to $86-88 \%$ minimums in subsequent cycles.

The practitioner reported warm internal feeling and intense perspiration starting between the fourth and sixth breathing cycle (4-6 min after the beginning of the exercise). This finding is consistent with previously reported studies [4][10], and could be explained by activation of VLF HRV band.

It was reported that very slow breathing is often characterized by a large number of arrhythmic events [4]. During our experiments only three recordings featured arrhythmic events (E01, E03, and E05) with 3, 1, and 4 events respectively. Unfortunately, we were not able to find out what was the main factor, and further research with more experiments is necessary. All events were located mostly at the beginning of the exercise, in the first several cycles. There are only two events located at 190s and 490s, the mean relative distance of the remaining six events was $74 \mathrm{~s}(\sigma=63 \mathrm{~s})$. Arrhythmic events didn't have significant influence on spectral characteristic. Some events might be contributed to the mechanical stretching of the SA node [11]. Most arrhythmic events were atrial extra-systoles generated during inhalation or exhalation, probably by overstimulation of the vagus. We hypothesize that the smaller number of arrhythmic events than reported in [4] could be explained by the breathing technique and practice.

In conclusion, we characterized basic statistical features of very slow breathing during Nadi Shodhana Pranayama as a valuable method of analyzing autonomous nervous system in extreme conditions. We have found the effect very similar to ANS resonance [3] with prominent heart rate oscillations [12]. However, we found that the resonance does not exist strictly at a single frequency but it changes 
dynamically, even for a single person, from session to session. We believe that previous studies of resonance of ANS didn't take into account available physiological margin of participants. Systematic practice gradually increases available physiological margin and establishes new resonant frequencies. One of the important unexpected findings of this study is similarity between physiological state immediately before and after the exercise. We believe the system starts establishment of relaxed hypo-metabolic state very quickly after assuming specific position for the practice, probably as a trained response.

We plan to measure continuous blood pressure to assess baroreflex sensitivity and develop a model that will characterize homeostatic changes during and after very slow yogic breathing exercise.

\section{ACKNOWLEDGMENTS}

We are grateful to Swami Devananda and Swami Niranjananda for insights into effects of pranayama techniques, Dr. Vladan Vukcevic for ECG analysis, Advanced Brain Monitoring and Vladislav Velimirovic for ARES data acquisition and $\mathrm{SpO} 2$ signal processing.

\section{REFERENCES}

[1] P. Zhang, W. Tapp, S. Reisman, B. Natelson, "Respiration Response Curve Analysis of Heart Rate Variability", IEEE Trans. Biomed Eng., April 1997, 44(4):321-325.
[2] H-S. Song, P.M. Lehrer, "The Effects of Specific Respiratory Rates on Heart Rate and Heart Rate Variability," Applied Psychophysiology and Biofeedback, March 2003, Vol. 28, No. 1, pp. 13-23.

[3] E. Vaschillo, P. Lehrer, N. Rishe, M. Konstantinov., "Heart rate variability biofeedback as a method for assessing baroreflex function: a preliminary study of resonance in the cardiovascular system," Appl Psychophysiol Biofeedback, March 2002, 27(1):1-27.

[4] P. Lehrer, Y. Sasaki, Y. Saito, "Zazen and Cardiac Variability," Psychosomatic Medicine, 61(6):812-821, 1999.

[5] G.C. Ray, A.Y. Kaplan, E. Jovanov, "Homeostatic Change in the Genesis of ECG During Yogic Breathing," Journal of the Institution of Engineers (India), Vol. 79, No. 1, March 1999, pp. 28-33.

[6] E. Jovanov, A. Lords, D. Raskovic, P. Cox, R. Adhami, F. Andrasik, "Stress Monitoring Using a Distributed Wireless Intelligent Sensor System," IEEE Engineering in Medicine and Biology Magazine, Vol. 22, No.3, May/June 2003, pp. 49-55.

[7] J. Mateo, P. Laguna, "Analysis of heart rate variability in the presence of ectopic beats using the heart timing signal," IEEE Trans Biomed Eng., March 2003, 50(3):334-43.

[8] Advanced Brain Monitoring, Apnea Risk Evaluation System (ARESTM), http://www.b-alert.com/ARES.html (October 2004).

[9] Swami Satyananda Saraswati, Asana Pranayama Mudra Bandha, Bihar School of Yoga, Bihar, India, 1996, ISBN 81-85787-14-X.

[10] H. Benson, J.W. Lehmann, M.S. Malhotra, R.F. Goldman, J. Hopkins, M.D. Epstein, "Body temperature changes during the practice of g Tum-mo yoga," Nature, Jan 21, 1982, 295(5846):234-6.

[11] E. Pyetan, S. Akselrod, "Do the high-frequency indexes of HRV provide a faithful assessment of cardiac vagal tone? A critical theoretical evaluation," IEEE Trans Biomed Eng, Jun 2003, 50(6):777-83.

[12] C.K. Peng, J.E. Mietus, Y. Liu, G. Khalsa, P.S. Douglas, H. Benson, A.L. Goldberger, "Exaggerated heart rate oscillations during two meditation techniques," Int J Cardiol, July 31, 1999, 70(2):101-7.

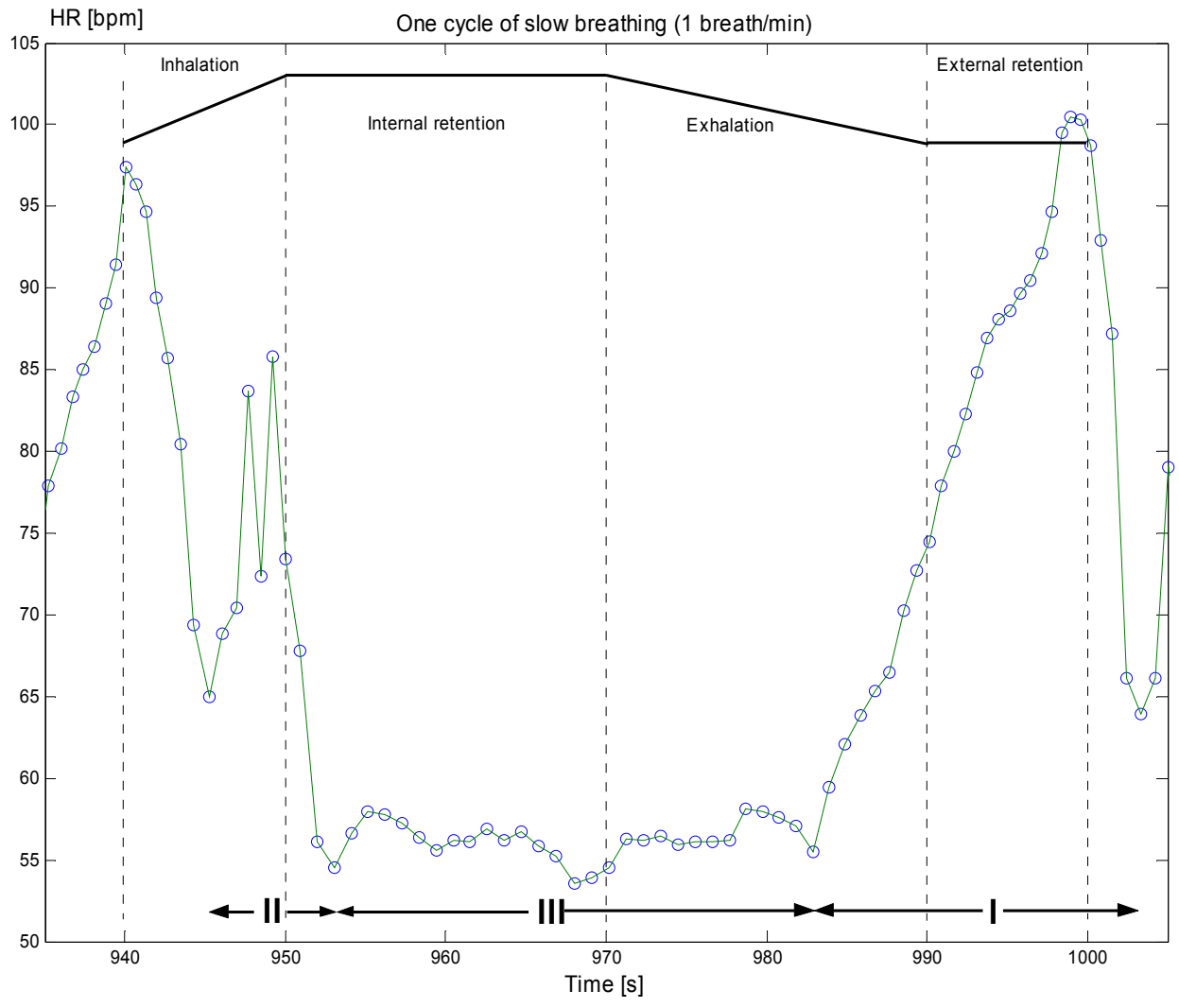

Fig. 2. Detailed diagram of one cycle of slow breathing with symbolic representation of breathing phases (file E01, $\mathrm{t}=940-1000 \mathrm{~s}$ ); Circles represent original heart beats. HRV phases: I) Abdominal exhalation and inhalation create drastic change in heart rate, II) thoracic and clavicular inhalation create increase in heart rate and HF oscillations, and III) internal retention, clavicular and thoracic exhalation generate very small LF oscillations. 
TABLE I

RESULTS OF STATISTICAL AND SPECTRAL ANALYSIS

\begin{tabular}{lrrr}
\hline \hline \multicolumn{1}{c}{ Parameter } & Before exercise & During exercise & After exercise \\
\hline Mean RRint (SE) & $959.29(10.26)$ & $904.13^{*}(11.81)$ & $998.98^{*}(14.45)$ \\
Total HRV power $\left[\mathrm{ms}^{2}\right]$ & $11,625.62$ & $9,036.96$ & $10,931.74$ \\
$(\mathrm{SE})$ & $(1,386.70)$ & $(836.64)$ & $(1,075.58)$ \\
VLF power $\left[\mathrm{ms}^{2}\right](\%)$ & $2,329.14(21.71)$ & $7,472.99^{*}\left(81.96^{*}\right)$ & $734.28^{*}\left(7.70^{*}\right)$ \\
LF power $\left[\mathrm{ms}^{2}\right](\%)$ & $8,494.94(70.54)$ & $1,352.76^{*}\left(15.80^{*}\right)$ & $9,823.10\left(88.65^{\star}\right)$ \\
HF power $\left[\mathrm{ms}^{2}\right](\%)$ & $801.54(7.75)$ & $211.21^{*}\left(2.24^{*}\right)$ & $374.36^{*}\left(3.64^{*}\right)$ \\
LF/HF (SE) & $14.33(3.73)$ & $9.50(1.80)$ & $50.93^{\dagger}(16.35)$ \\
LF max. frequency $[\mathrm{Hz}]$ & 0.091875 & 0.07525 & $0.07125^{*}$ \\
(SE) & $(0.00494)$ & $(0.00956)$ & $(0.00370)$ \\
HF max. frequency $[\mathrm{Hz}]$ & 0.174125 & 0.16 & 0.185625 \\
(SE) & $(0.00679)$ & $(0.00402)$ & $(0.01438)$ \\
\hline \hline
\end{tabular}

The results of raw HRV data processing; ectopic heart beats are not filtered out.

Significant differences when compared against the state before exercise: ${ }^{\dagger} p<0.05,{ }^{*} p<0.01,{ }^{*} p<0.001$ 